\title{
Pathophysiological Links Among Hypertension and Alzheimer's Disease
}

\author{
Daniela Carnevale $^{1,2} \cdot$ Marialuisa Perrotta $^{1} \cdot$ Giuseppe Lembo $^{1,2} \cdot$ Bruno $^{\text {Trimarco }}{ }^{3}$
}

Received: 13 April 2015/Accepted: 27 May 2015

(C) Springer International Publishing Switzerland 2015

\begin{abstract}
Genetic Alzheimer's disease (AD) accounts for only few $\mathrm{AD}$ cases and is almost exclusively associated to increased amyloid production in the brain. Instead, the majority of patients is affected with the $\mathrm{AD}$ sporadic form with typical alterations of clearance mechanisms of the brain. Most studies use engineered animal models that mimic genetic AD. Since it is emerging the existence of a pathophysiological link between cardiovascular risk factors and $\mathrm{AD}$ etiology, the strategy to develop animal models of vascular related $\mathrm{AD}$ pathology could be the key toward developing novel successful therapies. On this issue, we have demonstrated that mice that have been chronically subjected to high blood pressure show deposition of amyloid aggregates, the main histological feature of $\mathrm{AD}$, and loss of memory in specific tasks. More importantly, we have identified that the hypertensive challenge increases the expression of the receptor for advanced glycated end products (RAGE), leading to beta-amyloid (A $\beta$ ) deposition and learning impairment. Here, we review different murine models of hypertension, induced either pharmacologically or mechanically, leading in the long time to plaque formation in the brain parenchyma and around blood vessels. The major findings obtained till now in this particular experimental setting allow us to suggest that this appears to
\end{abstract}

Bruno Trimarco

trimarco@unina.it

1 Department of Angiocardioneurology and Translational Medicine, IRCCS Neuromed, Pozzilli, IS, Italy

2 Department of Molecular Medicine, Sapienza University of Rome, Rome, Italy

3 Division of Cardiology, Department of Advanced Biomedical Sciences, Federico II University, Naples, Italy be a unique possibility to study the pathogenetic mechanisms of sporadic AD triggered by vascular risk factors.

Keywords Alzheimer's disease (AD) - Hypertension and cardiovascular risk factors - Murine models of vascularrelated $\mathrm{AD}$ - Beta-amyloid $(\mathrm{A} \beta)$ deposition and cognitive impairment · RAGE, receptor for advanced glycated end products

\section{Introduction}

Hypertension has a key role in brain pathologies, such as stroke and dementia [1]. Lowering of elevated blood pressure is clearly an important therapeutic target in the prevention of stroke and, thus, of post stroke dementia [2]. Indeed, a single stroke can lead to dementia by interrupting neural connections involved in cognition and memory. The brain represents the main target organ of deleterious effects caused by hypertension, responsible of a large portion of related neurodegenerative disorders. Among these, Alzheimer's disease (AD) is considered the most common cause of dementia in elderly. A growing body of epidemiological studies have described that cardiovascular risk factors, such as hypertension, increases the onset of $\mathrm{AD}$ [3-5]. While early-onset/genetic $\mathrm{AD}$ accounts for only a small proportion of total cases and is associated to an increased production of the beta-amyloid $(\mathrm{A} \beta)$ peptides, the majority of patients is affected by late-onset/sporadic $A D$, due to a mechanism in which the $A \beta$ clearance is altered. Both vascular efflux of $A \beta$ across the blood-brain barrier (BBB) and/or influx of circulating $A \beta$ in the central nervous system (CNS) are mechanisms that, when dysregulated, may contribute to $A \beta$ aggregation and its deposition in brain parenchyma [4-6]. 
Chronic hypertension induces structural and functional changes in cerebral blood vessels strongly affecting the cerebral circulation. Recent findings have demonstrated that hypertension triggers hypoperfusion due to impairment of endothelial function that significantly modifies vascular compliance [1-7].

Studies on genetic AD animal models further suggest an interaction among anti-hypertensive therapies and a benefit on cerebral amyloid pathology $[8,9]$. However, the mechanism underlying this relation seems to be complex. The present review is aimed at providing novel insight into this issue. First, we will examine the cerebrovascular alterations induced by hypertension and then we will review the findings in a unique animal model available until now to study spontaneous $\mathrm{AD}$, focusing on the $\mathrm{AD}$-induced hypertension and developed in our laboratory during the last years.

\section{Vascular-Related Alzheimer Disease}

How do vascular factors increase the risk of AD? Although the answer to this question remains unclear, it represents one of the oldest issues in understanding relationships between $\mathrm{AD}$ and cerebrovascular diseases. The brain receives about $20 \%$ of cardiac output and the fine crosstalk between neurons and brain capillaries is critical for a proper brain functioning [1]. Indeed, when the cerebral blood flow (CBF) stops, neurons are damaged [10]. In AD the length reduction of brain capillaries can lead to a diminished transport of energy substrates and nutrients across the BBB $[11,12]$. The BBB is the brain endothelial structure specialized to separate components of the circulating blood from neurons and to maintain the chemical composition of the neuronal microenvironment [6]. Within pericytes, astrocytes and microglia, the BBB is required for preserving neuronal circuits and synaptic transmission. Hemodynamic changes caused by hypertension may disrupt the BBB, increasing the vascular permeability and responsible for the accumulation of $A \beta$ proteins in the cerebral parenchyma [13]. Soluble $A \beta$ monomers precipitate and deposit around neurons, forming insoluble and fibrillar aggregates $[14,15]$. These complex structures shape amyloid plaques characterizing $\mathrm{AD}[4]$ and lead to neuronal death in the hippocampus and cortex, responsible for cognitive decline and loss of memory [16]. Amyloid proteins have a different origin and their deposition in cerebral vasculature is still unknown; in fact, the formation of aggregates depends on the different maturation stage of peptides from monomeric to oligomeric forms, until complex fibrillar structure, which are the agents responsible of the early memory loss typical of $\mathrm{AD}[15,17]$. More important, the number of fragments correlates with the increase in blood pressure suggesting that pressure overload further aggravates $A \beta$ deposition in the brain.
Studies in endothelial cell cultures have suggest that peptides derived from APP (amyloid precursor protein) produce deleterious vascular effects that may contribute to neurodegeneration in AD. Since most cell types express APP and could potentially release A $\beta$, several mechanisms can lead to a different parenchymal plaque formation. An intriguing hypothesis poses that, the systemic mechanism whereby $A \beta$ is transferred from blood to the vasculature, could be related to the activation of RAGE, a major bidirectional transporter of $A \beta$ across the BBB [18]. In transgenic models of $\mathrm{AD}$, RAGE expression is increased in the affected cerebral vessels, microglia and neurons. In addition to RAGE, apolipoprotein $\mathrm{J}$ (apoJ) can facilitate transport of plasma-derived $\mathrm{A} \beta$ across the BBB [19]. LRP-1 (low density lipoprotein-receptor-related protein-1) mediates the opposite process, regulating the efflux from brain to circulation and is localized at the apical face of endothelium [20]. ApoE (apolipoprotein E), the most relevant risk factor associated with $\mathrm{AD}$, binds to LRP-1 to modulating the rate of $A \beta$ transport [19]. The exchange of $\mathrm{A} \beta$ between CNS, CNF and blood is an important process determining the concentration of $\mathrm{A} \beta$ in the brain. On this issue, the majority of patients affected by late-onset/sporadic $\mathrm{AD}$ may not have increased production of $\mathrm{A} \beta$ [6], but probably show a failure in the mechanisms responsible for the clearance of $A \beta$ from the brain.

\section{Murine Models of AD}

Brain hypoperfusion and impaired $\mathrm{BBB}$ clearance of $\mathrm{A} \beta$ lead to accumulation of neurotoxic $A \beta$ oligomers in the brain. Reduced blood flow and accumulated $A \beta$ oligomers can both initiate the neuronal injury. For this reason, over the last two decades, most attention has been focused on genetic animal models that could resemble the AD pathological features by overproducing the $A \beta$, resulting in its aggregation and deposition in brain parenchyma [21-24]. The disease phenotype includes the accumulation of $\mathrm{A} \beta$ and its deposition into senile plaques, the formation of tau containing tangles, reactive gliosis, inflammation and an immune response, neurodegeneration, cholinergic deficit, and cognitive impairment [25].

Transgenic mice are the most popular animal models for AD nowadays. The main reason behind this trend has been the development and application of novel molecular technologies, such as gene targeting, which enable researcher to engineer precise genetic alteration to study the neural basis of biochemical and behavioral alterations. The genetically modified models usually include: (i) Classical and conditional knockouts (KO). In the classical model, the function of the investigated gene is abolished from a very early stage of development, 
whereas, in conditional $\mathrm{KO}$ the gene inactivation is restricted either to a specific time window or brain region. (ii) Transgenics, in which a foreign gene is inserted into the genome; (iii) Knock-in, in which specific mutations are introduced in the gene leading to a loss of activity of the proteins encoded by the targeted gene (although the gene expression per se is not voided as it is in KOs). Genetic causes of AD are heterogeneous and include mutations or variants in several genes including the APP gene, the presenilin (PS), and ApoE [26]. The first successful genetically modified mouse models in $\mathrm{AD}$ research were transgenics for $A \beta$ by increasing the load of its precursor APP. In general, these lines develop robust amyloid pathology and show cognitive impairment. From a genetic point of view, all the genes encoding the secretases that cleave the APP molecule can be considered compelling candidate genes for AD. Especially interesting are the $\beta$ - and $\gamma$-secretases, which catalyze the processing of APP to the various A $\beta$ peptides [26], and $\alpha$-secretase, which is part of the non-amyloidogenic pathway [27]. ApoE mutation, which is strongest risk factor for $A D$, have also been used to produce mouse models of AD. In particular, the ApoE4 gene variant causes cognitive deficits whether expressed from astrocyte or in neuronal promoters [28]. On this issue, mice knock-in for ApoE4 display impairment in cognitive and synaptic plasticity as well as high plaque deposition when crossed with hAPP mice [29]. In mouse models of AD, including APPoverexpressing $\mathrm{APP}^{\mathrm{sw} / 0}$ mice and transgenic APP mice, dense plaques develop initially on blood vessels or as a classical CAA (cerebral amyloid angiopathy) [30]. It is believed that plaques are generated on blood vessels due to deficient $\mathrm{A} \beta$ clearance across the BBB. Drugs that downregulate RAGE and upregulate LRP at the BBB can readjust the transport equilibrium for $A \beta$ by promoting its efflux from brain into the bloodstream. In AD and in APP transgenic models of $\mathrm{AD}$, RAGE is significantly upregulated at the BBB, whereas LRP is downregulated [30].

However, the mouse models described above mimic genetic $\mathrm{AD}$, constituting only a small fraction of total $\mathrm{AD}$ cases. By contrast, our group has recently pursued the strategy to develop the only animal model of hypertensionrelated 'Alzheimer-like' pathology so far. In fact, we have found that mice that have been subjected to chronic high blood pressure showed accumulation of amyloid aggregates, the main histological finding of $\mathrm{AD}$, as well as other $\mathrm{AD}$ related features such as initiation of immune response, dysfunction of the BBB and cognitive impairment [31]. For a long time, a clear association between $\mathrm{AD}$ and hypertension has been reported, although the pathological link underlies such association were not completely understood. Much findings suggested that hypertension is an independent risk factor for the incidence and progression of brain pathology.

\section{Sporadic Versus Familial Alzheimer's Disease}

Alzheimer's Disease is the leading cause of dementia in elderly and, although some treatments help the symptoms and slow the progression of the disease, no definitive cure does exist. Early-onset/genetic AD accounts for only a small proportion of total cases and is associated to an increased production of the $A \beta$-amyloid peptides. On the contrary, the vast majority of patients is affected by late-onset/sporadic $\mathrm{AD}$, with no evident increase of $\mathrm{A} \beta$ production, but probably with a failure in the mechanisms responsible for clearance of $A \beta$ from the brain. Both vascular efflux of $A \beta$ across $B B B$ and influx of circulating $A \beta$ in the CNS are mechanisms that, when dysregulated, may contribute to $A \beta$ accumulation, leading to its aggregation and deposition [4-6].

Actually, it seems that the only step forward for development of novel rational therapies, is the identification of factors influencing onset/progression of the sporadic AD.

Despite the old belief that $\mathrm{AD}$, conversely to Vascular Dementia, has a non-vascular origin, a growing body of epidemiological studies strongly associate cardiovascular risk factors, such as hypertension, to increased incidence of $A D$ [6]. Indeed, it is now well known that hypertension exerts a strong impact on cerebral circulation, favoring hypoperfusion through endothelial function impairment, increased smooth muscle constrictor tone and structural remodeling that deeply modifies vascular compliance [1-7]. Moreover, recent studies on genetic $\mathrm{AD}$ animal models further suggest an interaction among anti-hypertensive therapies and a benefit on cerebral amyloid pathology and cognitive decline [8, 9]. However, despite these clear links between hypertension and $\mathrm{AD}$, few basic science studies has investigated this relationship so far.

\section{AD Induced by Hypertension: RAGE Hypothesis}

Many studies have focused on the possible mechanisms underlying the cognitive impairment hypertension-induced, but a pathophysiological link between hypertension and the loss of memory in specific brain areas is still missing. Cerebral blood vessels constitute the first line of defense for the brain from the peripheral challenges, like hypertension. Interestingly, RAGE receptor is expressed on endothelial cells, and we found that hypertensive challenge had an early effect of upregulation of RAGE expression in cognitive brain areas [32]. Moreover, a recent study examines the role of angiotensin II (AngII) type 1 receptor system for RAGE expression in cultured endothelial cells (ECs) from patients with essential hypertension [33]. AngII up-regulated RAGE mRNA levels of microvascular ECs and subsequently increased the expression of the soluble form of RAGE (sRAGE) in the medium of ECs, suggesting 
that the renin-angiotensin system (RAS) contributes to the development and progression of vascular angiopathy [33]. Chronic hypertension is associated with structural and functional changes in cerebral blood vessels and is crucial for the balance between CNS and periphery, maintained by BBB. Physiological equilibrium of the intracerebral and the bloodstream pools of $A \beta$ is due to specialized receptors that allow a tightly regulated shuttle across BBB [6]. The multiligand receptor RAGE, which binds also $A \beta / \beta$-sheet aggregates [34], dominate the influx at the BBB [21]. In view of these evidences, RAGE has been found up-regulated in human $\mathrm{AD}$ brains [35], has a pivotal role in brain $\mathrm{A} \beta$ deposition in $\mathrm{AD}$ experimental models [21], and has been proposed as a fundamental mechanism signaling danger to vulnerable vasculature [36]. Moreover, we demonstrated that high blood pressure might interfere with RAGE signaling inducing the AD pathology. On this issue, we have exploited our murine model of hypertension-induced AD-related pathology [31-37], to investigate the role of RAGE. Interestingly, mice with genetic ablation of RAGE, showed protection from hypertension-induced $A \beta$ deposition and cognitive deterioration. [32]. Interestingly, we obtained a similar protection when we used a recently developed high-affinity tertiary amide RAGE-specific inhibitor (FPS-ZM1), shown to block A $\beta$ binding specifically to the V-domain of RAGE [38], thus opening novel therapeutic opportunities for vascular forms of AD.

\section{Conclusions}

Despite the established clinical link between hypertension and $\mathrm{AD}$, only a few basic science studies have investigated the specific molecular relationship so far. In particular, while most studies use engineered animal models that mimic genetic $\mathrm{AD}$, constituting only a small fraction of $\mathrm{AD}$ cases, we pursued the strategy to exploit vascular related animal models. On this issue, the possibility to manipulate vascular risk factors, like hypertension, in mice, in order to investigate the impact on brain pathology and cognitive deficits ascribable to $\mathrm{AD}$, appears as the best chance to allow investigators to dissect the molecular mechanisms of spontaneous $\mathrm{AD}$ and thus afford novel and targeted therapeutic strategies.

\section{References}

1. Iadecola C, Davisson RL. Hypertension and cerebrovascular dysfunction. Cell Metab. 2008;7:476-84.

2. Lammie GA. Hypertensive cerebral small vessel disease and stroke. Brain Pathol. 2002;12:358-70.

3. Skoog I, Gustafson D. Update on hypertension and Alzheimer's disease. Neurol Res. 2006;28:605-11.

4. De la Torre JC. Alzheimer disease as a vascular disorder: nosological evidence. Stroke. 2003;33:1152-62.
5. Fotuhi M, Hachinski V, Whitehouse PJ. Changing perspectives regarding late-life dementia. Nat Rev Neurol. 2009;5:649-58.

6. Zlokovic BV. The blood-brain barrier in health and chronic neurodegenerative disorders. Neuron. 2008;57:178-201.

7. Hainsworth AH, Markus HS. Do in vivo experimental models reflect human cerebral small vessel disease? A systematic review. J Cereb Blood Flow Metab. 2008;28:1877-9.

8. Tsukuda K, Mogi M, Iwanami J, Min LJ, Sakata A, Jing F, Iwai M, Horiuchi M. Cognitive deficit in amyloid- $\beta$-injected mice was improved by pretreatment with a low dose of telmisartan partly because of peroxisome proliferator-activated receptor- $\gamma$ activation. Hypertension. 2009;54:782-7.

9. Wang J, Ho L, Chen L, Zhao Z, Zhao W, Qian X, Humala N, Seror I, Bartholomew S, Rosendorff C, Pasinetti GM. Valsartan lowers brain $\beta$-amyloid protein levels and improves spatial learning in a mouse model of Alzheimer disease. J Clin Invest. 2007;117:3393-402.

10. Girouard H, Park L, Anrathe J, Zhou P, Iadecola C. Cerebrovascular nitrosative stress mediates neurovascular and endothelial dysfunction induced by angiotensin II. Arterioscler Thromb Vasc Biol. 2006;27:303-9.

11. Bailey TL, Rivara CB, Rocher AB, Hof PR. The nature and effects of cortical microvascular pathology in aging and Alzheimer's disease. Neurol Res. 2004;26:573-8.

12. Wu Z, Guo H, Chow N, Sallstrom J, Bell RD, Deane R, Brooks AI, Kanagala S, Rubio A, Sagare A, et al. Role of the MEOX2 homeobox gene in neurovascular dysfunction in Alzheimer disease. Nat Med. 2005;11:959-65.

13. Thomas T, Thomas G, McLendon C, Sutton T, Mullan M. betaAmyloid-mediated vasoactivity and vascular endothelial damage. Nature. 1996;380:168-71.

14. Selkoe DJ. Soluble oligomers of the amyloid beta-protein impair synaptic plasticity and behavior. Behav Brain Res. 2008;192:106-13.

15. Haass C, Selkoe DJ. Soluble protein oligomers in neurodegeneration: lessons from the Alzheimer's amyloid $\beta$-peptide. Nat Rev Mol Cell Biol. 2007;8:101-12.

16. Perlmutter LS, Barron E, Saperia D, Chui H. Association between vascular basement membrane components and the lesions of Alzheimer's disease. J Neurosci Res. 1991;30:673-81.

17. Lesné S, Koh MT, Kotilinek L, Kayed R, Glabe CG, Yang A, Gallagher M, Ashe KH. A specific amyloid-beta protein assembly in the brain impairs memory. Nature. 2006;440:352-7.

18. Deane R, Du Yan S, Submamaryan RK, LaRue B, Jovanovic S, Hogg E, Welch D, Manness L, Lin C, Yu J, Zhu H, Ghiso J, Frangione B, Stern A, Schmidt AM, Armstrong DL, Arnold B, Liliensiek B, Nawroth P, Hofman F, Kindy M, Stern D, Zlokovic B. RAGE mediates amyloid-peptide transport across the bloodbrain barrier and accumulation in brain. Nat Med. 2003;9:907-13.

19. Zlokovic BV, Ghiso J, Mackic JB, McComb JG, Weiss MH, Frangione B. Blood-brain barrier transport of circulating Alzheimer's amyloid $\beta$. Biochem Biophys Res Commun. 1993; 197:1034-40.

20. Mackic JB, Weiss MH, Miao W, Kirkman E, Ghiso J, Calero M, Bading J, Frangione B, Zlokovic BV. Cerebrovascular accumulation and increased blood-brain barrier permeability to circulating Alzheimer's amyloid $\beta$ peptide in aged squirrel monkey with cerebral amyloid angiopathy. J Neurochem. 1998;70:210-5.

21. Hardy J, Selkoe DJ. The amyloid hypothesis of Alzheimer's disease: progress and problems on the road to therapeutics. Science. 2002;297:353-6.

22. Scheuner D, Eckman C, Jensen M, Song X, Citron M, Suzuki N, Bird TD, Hardy J, Hutton M, Kukull W, et al. Secreted amyloid $\beta$-protein similar to that in the senile plaques of Alzheimer's disease is increased in vivo by the presenilin 1 and 2 and APP 
mutations linked to familial Alzheimer's disease. Nat Med. 1996;2:864-70.

23. Sterniczuk R, Dyck RH, Laferla FM, Antle MC. Characterization of the 3xTg-AD mouse model of Alzheimer's disease. Part 1. Circadian changes. Brain Res. 2010;1348:139-48.

24. Sterniczuk R, Antle MC, Laferla FM, Dyck RH. Characterization of the 3xTg-AD mouse model of Alzheimer's disease. Part 2. Behavioral and cognitive changes. Brain Res. 2010;1348:149-55.

25. Mattson MP. Pathways towards and away from Alzheimer's disease. Nature. 2004;430:631-9.

26. Kobayashi DT, Chen KS. Behavioral phenotypes of amyloidbased genetically modified mouse models of Alzheimer's disease. Genes Brain Behav. 2005;4:173-96.

27. Lammich S, Kojro E, Postina R, Gilbert S, Pfeiffer R, Jasionowski M, Haass C, Fahrenholz F. Constitutive and regulated alpha-secretase cleavage of Alzheimer's amyloid precursor protein by a disintegrin metalloprotease. Prot Natl Acad Sci. 1999;96:3922-7.

28. Hartman RE, Wozniak DF, Nardi A, Olney JW, Sartorius L, Holtzman DM. Behavioral phenotyping of GFAP-apoE3 and apoE4 transgenic mice: apoE4 mice show profound working memory impairments in the absence of Alzheimer's-like neuropathology. Exp Neurol. 2001;170:326-44.

29. Sullivan PM, Han B, Liu F, Mace BE, Ervin JF, Wu S, Koger D, Paul S, Bales KR. Reduced levels of human apoE4 protein in an animal model of cognitive impairment. Neurobiol Aging. 2009;32:791-801.

30. Deane R, Wu Z, Zlokovic BV. RAGE (yin) versus LRP (yang) balance regulates alzheimer amyloid beta-peptide clearance through transport across the blood-brain barrier. Stroke. 2004;35:2628-31.

31. Carnevale C, Mascio G, Ajmone-Cat MA, D’Andrea I, Cifelli G, Madonna M, Cocozza G, Frati A, Carullo P, Carnevale L, Alleva E, Branchi I, Lembo G, Minghetti L. Role of neuroinflammation in hypertension-induced brain amyloid pathology. Neurobiol Aging. 2012;33:205.e19-29.

32. Carnevale D, Mascio G, D'Andrea I, Fardella V, Bell RD, Branchi I, Pallante F, Zlokovic B, Yan SS, Lembo G. Hypertension induces brain $\beta$-amyloid accumulation, cognitive impairment, and memory deterioration through activation of receptor for advanced glycation end products in brain vasculature. Hypertension. 2012;60:188-97.

33. Nakamura K, Yamagishi S, Nakamure Y, Takenaka K, Matsui T, Jinnouchi Y, Imaizumi T. Telmisartan inhibits expression of a receptor for advanced glycation end products (RAGE) in angiotensin-II-exposed endothelial cells and decreases serum levels of soluble RAGE in patients with essential hypertension. Microvasc Res. 2005;70:137-41.

34. Bierhaus A, Nawroth PP. Posttranslational modification of lipoproteins-a fatal attraction in metabolic disease? J Alzheimers Dis. 2005;7:315-7.

35. Donahue JE, Flaherty SL, Johanson CE, Duncan JA, Silverberg GD, Miller MC, Tavares R, Yang W, Wu Q, Sabo E, Hovanesian V, Stopa EG. RAGE, LRP-1, and amyloid-beta protein in Alzheimer's disease. Acta Neuropathol. 2006;112:405-15.

36. Yan SF, Ramasamy R, Schmidt AM. The RAGE axis: a fundamental mechanism signaling danger to the vulnerable vasculature. Circ Res. 2010;106:842-53.

37. Gentile MT, Poulet R, Di Pardo A, Cifelli G, Maffei A, Vecchione C, Passarelli F, Landolfi A, Carullo P, Lembo G. $\beta$ Amyloid deposition in brain is enhanced in mouse models of arterial hypertension. Neurobiol Aging. 2009;30:222-8.

38. Deane R, Singh I, Sagare AP, Bell RD, Ross NT, LaRue B, Love R, Perry S, Paquette N, Deane RJ, Meenakshisundaram T, Zarcone T, Fritz G, Friedeman AE, Miller BL, Zlokovic BV. A multimodal RAGE-specific inhibitor reduces amyloid $\beta$-mediated brain disorder in a mouse model of Alzheimer disease. J Clin Invest. 2012;122:1377-92. 